\title{
GENERIC SINGULAR SPECTRUM FOR ERGODIC SCHRÖDINGER OPERATORS
}

\section{ARTUR AVILA and DAVID DAMANIK}

\begin{abstract}
We consider Schrödinger operators with ergodic potential $V_{\omega}(n)=f\left(T^{n}(\omega)\right), n \in \mathbb{Z}$, $\omega \in \Omega$, where $T: \Omega \rightarrow \Omega$ is a nonperiodic homeomorphism. We show that for generic $f \in C(\Omega)$, the spectrum has no absolutely continuous component. The proof is based on approximation by discontinuous potentials which can be treated via Kotani theory.
\end{abstract}

\section{Introduction}

Let $\Omega$ be a compact metric space, let $T: \Omega \rightarrow \Omega$ be a homeomorphism, and let $\mu$ be a $T$-ergodic Borel probability measure. We always assume that $T$ is not periodic; that is, $\mu$ is nonatomic. For a bounded and measurable function $f: \Omega \rightarrow \mathbb{R}$, we consider (line) Schrödinger operators $H_{\omega}=\Delta+V_{\omega}, n \in \mathbb{Z}$, with potential $V_{\omega}(n)=f\left(T^{n} \omega\right)$ and the associated Lyapunov exponents $\gamma(z), z \in \mathbb{C}$. By Kunz and Souillard [5] (see also [13]), there exists a compact set $\Sigma_{\mathrm{ac}}(f) \subseteq \mathbb{R}$ such that $\sigma_{\mathrm{ac}}\left(H_{\omega}\right)=\Sigma_{\mathrm{ac}}(f)$ for $\mu$-a.e. $\omega \in \Omega$. By Pastur [15], Ishii [10], and Kotani [11] (see also [5], [16]), $\Sigma_{\mathrm{ac}}(f)=\overline{\{E \in \mathbb{R}: \gamma(E)=0\}}{ }^{\mathrm{ess}}$.

We consider only situations where the potentials $V_{\omega}$ are not periodic. In this case, it is an interesting question whether there can be any absolutely continuous spectrum.

It was observed by Kotani [12] that $\Sigma_{\text {ac }}(f)$ is empty if $f$ takes only finitely many values. Damanik and Killip [6] derived the same conclusion under the assumption that $f$ is discontinuous at some point $\omega_{0}$ (but continuous at all points in the forward orbit of $\omega_{0}$ under $T$ ). Here we consider the case of continuous $f$. It is not in general true in this case that $\Sigma_{\mathrm{ac}}(f)$ is empty; if $\Omega=\mathbb{R} / \mathbb{Z}, T$ is a Diophantine irrational rotation, $v$ is analytic, and $\lambda>0$ is small enough, then for $f=\lambda v$, the spectrum is (almost surely) purely absolutely continuous (see, e.g., Bourgain and Jitomirskaya [4] and references therein). However, we show that absence of absolutely continuous spectrum is a generic phenomenon.

\section{THEOREM 1}

There is a residual set of functions $f$ in $C(\Omega)$ such that $\Sigma_{\mathrm{ac}}(f)=\emptyset$.

DUKE MATHEMATICAL JOURNAL

Vol. 130, No. 2, (C) 2005

Received 3 September 2004. Revision received 24 February 2005.

2000 Mathematics Subject Classification. Primary 82B44; Secondary 47B36.

Damanik's work supported in part by National Science Foundation grant DMS-0227289. 


\section{Remark 1.1}

A subset of a complete metric space (or, more generally, a Baire space) is called residual or (Baire) generic if it contains a countable intersection of dense open sets. By Baire's theorem, such a set is dense.

\section{Remark 1.2}

It is well known (see [3] for a more general result) that for generic potentials, the Lyapunov exponent vanishes generically in the spectrum. Frequently (e.g., if $T$ : $\Omega \rightarrow \Omega$ has a periodic point in the support of $\mu$ ), the spectrum contains intervals in a persistent way and hence has positive Lebesgue measure. This is not incompatible with our result since the Lyapunov exponent can be discontinuous.

The method used in the proof of Theorem 1 can be applied to certain one-parameter families of potentials. This has the following consequence.

\section{THEOREM 2}

There is a residual set of functions $f$ in $C(\Omega)$ such that $\Sigma_{\mathrm{ac}}(\lambda f)=\emptyset$ for almost every $\lambda>0$.

This is particularly striking in the case of quasi-periodic systems. In this context, one often expects absolutely continuous spectrum for small coupling, and as we mentioned before, this has been established under strong regularity assumptions on the potential (see Bourgain and Jitomirskaya's result [4]). It was not clear whether the assumption on the regularity was an artifact of the known methods. Our result shows that some regularity is indeed necessary; it is not enough to assume only continuity of the potential.

It is an interesting open problem to determine the weakest possible regularity assumption on $f$ under which a Bourgain-Jitomirskaya-type result holds. In this context, we note that Theorems 1 and 2 fail in the sufficiently differentiable case. For instance, it follows from Kolmogorov-Arnold-Moser (KAM) arguments that if $T$ is a Diophantine irrational rotation of $\Omega=\mathbb{R}^{d} / \mathbb{Z}^{d}$, then there exist $r<\infty$ and $\varepsilon>0$ such that if $f$ is $C^{r}$ and $\|f\|_{C^{r}}<\varepsilon$, then $\Sigma_{\mathrm{ac}}(f)$ is nonempty.*

\section{A semicontinuity result}

We need some basic facts about Lyapunov exponents and $m$-functions (see [16]). The Lyapunov exponent is defined by

$$
\gamma_{f}(E)=\lim _{n \rightarrow \infty} \frac{1}{n} \int_{\Omega} \ln \left\|S_{f, E}^{n}(\omega)\right\| d \mu(\omega),
$$

${ }^{*}$ In the case where $\Omega=\mathbb{R} / \mathbb{Z}, T$ the golden-mean rotation, a direct use of the Arnold-Moser theorem as stated in [9, Theorem B.2.2], gives $r=4+\epsilon$ for every $\epsilon>0$, but this is probably not optimal for our problem. 
where

$$
S_{f, E}^{n}(\omega)=S_{f, E}\left(T^{n-1}(\omega)\right) \cdots S_{f, E}(\omega)
$$

and

$$
S_{f, E}(\omega)=\left(\begin{array}{cc}
E-f(\omega) & -1 \\
1 & 0
\end{array}\right) \in \operatorname{SL}(2, \mathbb{C}) .
$$

We have that $E \mapsto \gamma_{f}$ is a nonnegative real-symmetric subharmonic function. If

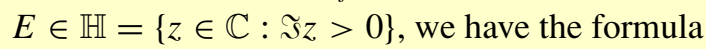

$$
\gamma_{f}(E)=\int_{\Omega}-\Re \ln m_{\omega, f}(E) d \mu(\omega),
$$

where $m_{\omega, f}: \mathbb{H} \rightarrow \mathbb{H}$ is a holomorphic function called the $m$-function which is given almost everywhere by

$$
m_{\omega, f}(E)=\lim _{n \rightarrow \infty} S_{f, E}^{n}\left(T^{-n}(\omega)\right) \cdot i
$$

(Here we consider the usual action of $\operatorname{SL}(2, \mathbb{C})$ by Möbius transformations, i.e., $\left(\begin{array}{ll}a & b \\ c & d\end{array}\right) \cdot z=(a z+b) /(c z+d)$. $)$

Define $M(f)=\left|\left\{E \in \mathbb{R}: \gamma_{f}(E)=0\right\}\right|$, where $|\cdot|$ denotes Lebesgue measure. As a consequence of the results of Ishii, Kotani, and Pastur, we have $\Sigma_{\mathrm{ac}}(f)=\emptyset$ if and only if $M(f)=0$.

LEMMA 1

For every $r>0, \Lambda>0$, the maps

$$
\left(L^{1}(\Omega) \cap B_{r}\left(L^{\infty}(\Omega)\right),\|\cdot\|_{1}\right) \rightarrow \mathbb{R}, \quad f \mapsto M(f),
$$

and

$$
\left(L^{1}(\Omega) \cap B_{r}\left(L^{\infty}(\Omega)\right),\|\cdot\|_{1}\right) \rightarrow \mathbb{R}, \quad f \mapsto \int_{0}^{\Lambda} M(\lambda f) d \lambda,
$$

are upper semicontinuous.

\section{Proof}

It is enough to show that (1) is upper semicontinuous since this implies that (2) is also upper semicontinuous by Fatou's lemma.

We have to show that if $\left(f_{n}\right)_{n \in \mathbb{Z}_{+}}, f$ are uniformly bounded in $L^{\infty}$ and $f_{n} \rightarrow f$ in $L^{1}$, then $\lim \sup M\left(f_{n}\right) \leq M(f)$.

Assume otherwise. Then (by passing to a suitable subsequence) there are a constant $C<\infty$ and a sequence $\left(f_{n}\right)$ such that 
(i) $\quad f_{n} \rightarrow f$ in $L^{1}$ and pointwise,

(ii) $\left\|f_{n}\right\|_{\infty} \leq C,\|f\|_{\infty} \leq C$,

(iii) $\liminf M\left(f_{n}\right) \geq M(f)+\varepsilon$ for some $\varepsilon>0$.

By (i), we have pointwise convergence of the $m$-functions $m_{\omega, f_{n}}$ in $\mathbb{H}$ for almost every $\omega$. Thus, by dominated convergence and (ii), the associated Lyapunov exponents $\gamma_{f_{n}}(E)$ converge pointwise in $\mathbb{H}$ to $\gamma_{f}(E)$.

By (ii), all Lyapunov exponents are positive outside the interval $I=[-2-$ $C, 2+C]$. Thus, we can limit our attention to this interval. Consider the region $U$ in $\mathbb{H}$, bounded by the equilateral triangle $T$ with sides $I, J, K$. Consider a conformal mapping $\Phi$ from the unit disk $\mathbb{D}$ to $U$. By the Schwarz-Christoffel formula (see, e.g., [8]),

$$
\Phi^{\prime}(z)=\text { const } \cdot \prod_{j=1}^{3}\left(1-\frac{z}{z_{j}}\right)^{-2 / 3}
$$

where $z_{1}, z_{2}, z_{3}$ are the inverse images under $\Phi$ of the vertices of $T$.

The functions $\gamma_{f_{n}} \circ \Phi$ are harmonic and bounded in $\mathbb{D}$. This yields

$$
\gamma_{f_{n}}(\Phi(0))=\frac{1}{2 \pi} \int_{0}^{2 \pi} \gamma_{f_{n}}\left(\Phi\left(e^{i \theta}\right)\right) d \theta,
$$

and similarly for $\gamma_{f}$. Since $\gamma_{f_{n}}(\Phi(0)) \rightarrow \gamma_{f}(\Phi(0))$ as $n \rightarrow \infty$, we infer

$$
\frac{1}{2 \pi} \int_{0}^{2 \pi}\left[\gamma_{f_{n}}\left(\Phi\left(e^{i \theta}\right)\right)-\gamma_{f}\left(\Phi\left(e^{i \theta}\right)\right)\right] d \theta \rightarrow 0 .
$$

By dominated convergence, the integrals along $J$ and $K$ go to zero individually. Therefore,

$$
\int_{I}\left[\gamma_{f_{n}}(E)-\gamma_{f}(E)\right] g(E) d E \rightarrow 0,
$$

where $g(E)=\left[\Phi^{\prime}\left(\Phi^{-1}(E)\right)\right]^{-1}$. It follows from (3) that $g$ vanishes at the endpoints of $I$ and is continuous and nonvanishing inside $I$.

By upper semicontinuity of the Lyapunov exponent and dominated convergence,

$$
\int_{I} \max \left\{\gamma_{f_{n}}(E)-\gamma_{f}(E), 0\right\} g(E) d E \rightarrow 0,
$$

and hence

$$
\int_{I} \min \left\{\gamma_{f_{n}}(E)-\gamma_{f}(E), 0\right\} g(E) d E \rightarrow 0
$$


Consequently, since $\gamma_{f} \mid I$ is bounded and $\gamma_{f_{n}} \mid I$ is nonnegative,

$$
\int_{I} \min \left\{\gamma_{f_{n}}(E)-\gamma_{f}(E), 0\right\} d E \rightarrow 0 .
$$

Choose $\delta>0$ such that the set $X=\left\{E \in I: \gamma_{f}(E)<\delta\right\}$ has measure bounded by $M(f)+\varepsilon / 4$ with $\varepsilon$ from (iii). Then

$$
\int_{I \backslash X} \min \left\{\gamma_{f_{n}}(E)-\gamma_{f}(E), 0\right\} d E \rightarrow 0 .
$$

This shows that for $n \geq n_{0}$, there exists a set $Y_{n}$ of measure bounded by $\varepsilon / 4$ such that $\gamma_{f_{n}}(E) \geq \delta / 2$ for every $E \in I \backslash\left(X \cup Y_{n}\right)$. Consequently, $\lim \sup M\left(f_{n}\right) \leq M(f)+\varepsilon / 2$, which contradicts (iii).

\section{Approximation by discontinuous potentials}

\section{LEMMA 2}

There exists a dense subset $\mathscr{Z}$ of $L^{\infty}(\Omega)$ such that if $s \in Z$, then

(1) $s(\omega), \omega \in \Omega$, takes finitely many values;

(2) $\quad s\left(T^{n}(\omega)\right), n \in \mathbb{Z}$, is not periodic for almost every $\omega \in \Omega$.

\section{Proof}

Let $W_{k}$ be the closed subspace of functions $s$ taking at most $k$ values. Obviously, $W=\bigcup_{k \geq 2} W_{k}$ is dense in $L^{\infty}(\Omega)$. So we only have to show that there is a dense subset $S_{k} \subset W_{k}$ of functions satisfying the second property. Given $s \in W, \omega \in \Omega$, let $\phi(s, \omega) \in \mathbb{Z}_{+} \cup\{\infty\}$ be the period of $s\left(T^{n}(\omega)\right), n \in \mathbb{Z}$. Then $\phi(s, \omega)$ is a constant $\Phi(s)$ almost everywhere. Let $W_{k, n}=\left\{s \in W_{k}: \Phi(s) \leq n\right\}$. It is easy to see that $W_{k, n}$ is a closed subset of $W_{k}$ and $W_{k} \neq W_{k, n}$. Thus, $W_{k} \backslash \bigcup_{n \in \mathbb{Z}_{+}} W_{k, n}$ is dense in $W_{k}$.

\section{LEMMA 3}

For $f \in C(\Omega), \varepsilon>0, \delta>0, \Lambda>0$, there exists $\tilde{f} \in C(\Omega)$ such that $\|f-\tilde{f}\|_{\infty}<\varepsilon$, $M(\tilde{f})<\delta$, and $\int_{0}^{\Lambda} M(\lambda \tilde{f}) d \lambda<\delta$.

\section{Proof}

Let $\mathscr{Z}$ be as in Lemma 2, and choose $s \in \mathscr{Z}$ such that $\|f-s\|_{\infty}<\varepsilon / 2$. By the Kotani result [12], we have $M(\lambda s)=0$ for every $\lambda>0$. Next we choose continuous functions $f_{n}$, for which we have $\left\|s-f_{n}\right\|_{\infty}<\varepsilon / 2$ for all $n$ and $\left\|s-f_{n}\right\|_{1} \rightarrow 0$ as $n \rightarrow \infty$. For instance, take $f_{n}(\omega)=\int_{\Omega} C_{n}(\omega)^{-1} c_{n}\left(\omega, \omega^{\prime}\right) s\left(\omega^{\prime}\right) d \nu\left(\omega^{\prime}\right)$, where $v$ is a probability measure with $\operatorname{supp} v=\Omega, C_{n}(\omega)=\int_{\Omega} c_{n}\left(\omega, \omega^{\prime}\right) d v\left(\omega^{\prime}\right)$, $c_{n}\left(\omega, \omega^{\prime}\right)=\max \left\{\left(n+n_{0}\right)^{-1}-\operatorname{dist}\left(\omega, \omega^{\prime}\right), 0\right\}$, and $n_{0}$ sufficiently large. Lemma 1 
implies $M\left(f_{n}\right), \int_{0}^{\Lambda} M\left(\lambda f_{n}\right) d \lambda \rightarrow 0$ as $n \rightarrow \infty$. Thus, choosing $n$ large enough so that $M\left(f_{n}\right), \int_{0}^{\Lambda} M\left(\lambda f_{n}\right) d \lambda<\delta$, we complete the proof.

Proof of Theorem 1

For $\delta>0$, we define

$$
M_{\delta}=\{f \in C(\Omega): M(f)<\delta\} .
$$

By Lemma $1, M_{\delta}$ is open, and by Lemma $3, M_{\delta}$ is dense. It follows that

$$
\left\{f \in C(\Omega): \Sigma_{\mathrm{ac}}(f)=\emptyset\right\}=\{f \in C(\Omega): M(f)=0\}=\bigcap_{\delta>0} M_{\delta}
$$

is residual.

Proof of Theorem 2

For $\Lambda, \delta>0$, we define

$$
M_{\delta}(\Lambda)=\left\{f \in C(\Omega): \int_{0}^{\Lambda} M(\lambda f) d \lambda<\delta\right\} .
$$

By Lemma $1, M_{\delta}(\Lambda)$ is open, and by Lemma $3, M_{\delta}(\Lambda)$ is dense. Thus,

$$
\bigcap_{\Lambda, \delta>0} M_{\delta}(\Lambda)
$$

is residual. It follows that for Baire generic $f \in C(\Omega)$, we have $\Sigma_{\mathrm{ac}}(\lambda f)=\emptyset$ for almost every $\lambda>0$.

\section{Concluding remarks}

\section{Remark 4.1}

It is possible to improve Lemma 1 to show that $M(f)$ is an upper semicontinuous function of $f \in L^{1}(\Omega)$. The additional point is that, given $f \in L^{1}(\Omega)$ and $\varepsilon>0$, we can choose a bounded interval $I \subset \mathbb{R}$ such that for every $\tilde{f} \in L^{1}(\Omega)$ close to $f$, we have $\left|\left\{E \in \mathbb{R} \backslash I: \gamma_{\tilde{f}}(E)=0\right\}\right|<\varepsilon$. To see this, first one shows that the integrated density of states $N_{f}(E) \in L^{\infty}(\mathbb{R})$ is a continuous function of $f \in L^{1}(\Omega)$, and then one uses [7] to bound the size of the absolutely continuous spectrum near infinity.

\section{Remark 4.2}

By the Wonderland theorem [17, p. 137] (see also [14]), the set of $f$ 's leading to purely singular spectrum is a $G_{\delta}$-set in all metric topologies that imply strong resolvent convergence of the associated operators. This permits one to deduce generic singular 
spectrum if one can exhibit a dense set with this property. With the Kotani result (combined with Lemma 2) as input, this gives only a generic set in $L^{\infty}(\Omega)$ and does not imply Theorem 1. It is not clear how to prove Theorem 1 using this strategy, but it would be interesting to find an explicit dense set of continuous functions such that the corresponding operators have empty absolutely continuous spectrum.

\section{Remark 4.3}

The result of this article naturally extends to the context of more general $\operatorname{SL}(2, \mathbb{R})$-cocycles. A possible formulation is the following. Given $A \in C(\Omega$, $\operatorname{SL}(2, \mathbb{R}))$, one can consider a one-parameter family of cocycles $\left(T, R_{\theta} A\right)$, where $R_{\theta}=\left(\begin{array}{rr}\cos \theta & -\sin \theta \\ \sin \theta & \cos \theta\end{array}\right)$, and the result is that for generic $A$ and for almost every $\theta \in \mathbb{R}$, the Lyapunov exponent of $\left(T, R_{\theta} A\right)$ is positive. The key point is that the relevant part of Kotani's theory (which is used in the proof of Lemma 3) can be carried out in this setting (see [2] for related results). (To prove the analogue of Lemma 1, one can use [1] to show that the average Lyapunov exponent of the family $\theta \mapsto\left(T, R_{\theta} A\right)$ depends continuously on $A$ in the $L^{1}$-topology.)

Notice that for certain choices of $T$ (say, irrational rotations), there are open sets $U \subset C(\Omega, \operatorname{SL}(2, \mathbb{R}))$ such that the Lyapunov exponent of $\left(T, R_{\theta} A\right)$ is zero for generic $A \in U$ and for generic $\theta \in \mathbb{R}$ (see [3]). Based on this, some authors have argued that under weak smoothness requirements (such as continuity), positive Lyapunov exponents are rare. Our result shows in a sense that positive Lyapunov exponents tend to prevail in a mixed topological/measure-theoretic category, even when they are topologically rare.

Acknowledgments. This work was done while Avila was visiting the California Institute of Technology. We thank Svetlana Jitomirskaya and Barry Simon for stimulating discussions.

\section{References}

[1] A. AVILA and J. BOCHI, A formula with some applications to the theory of Lyapunov exponents, Israel J. Math. 131 (2002), 125-137. MR 1942304399

[2] A. AVILA and R. KRIKORIAN, Quasiperiodic $\operatorname{SL}(2, \mathbb{R})$ cocycles, in preparation. 399

[3] J. BOCHI, Genericity of zero Lyapunov exponents, Ergodic Theory Dynam. Systems 22 (2002), 1667-1696. MR 1944399 394, 399

[4] J. BOURGAIN and S. JITOMIRSKAYA, Absolutely continuous spectrum for $1 D$ quasiperiodic operators, Invent. Math. 148 (2002), 453-463. MR 1908056 393,394 
[5] H. L. CYCON, R. G. FROESE, W. KIRSCH, and B. SIMON, Schrödinger Operators with Application to Quantum Mechanics and Global Geometry, Texts Monogr. Phys., Springer, Berlin, 1987. MR 0883643393

[6] D. DAMANIK and R. KILLIP, Ergodic potentials with a discontinuous sampling function are nondeterministic, Math. Res. Lett. 12 (2005), 187-192. MR 2150875393

[7] P. DEIFT and B. SIMON, Almost periodic Schrödinger operators, III: The absolutely continuous spectrum in one dimension, Comm. Math. Phys. 90 (1983), 389-411. MR 0719297398

[8] T. A. DRISCOLL and L. N. TREFETHEN, Schwarz-Christoffel Mapping, Cambridge Monogr. Appl. Comput. Math. 8, Cambridge Univ. Press, Cambridge, 2002. MR 1908657396

[9] M.-R. HERMAN, Sur la conjugaison différentiable des difféomorphismes du cercle à des rotations, Inst. Hautes Études Sci. Publ. Math. 49 (1979), 5-233. MR 0538680394

[10] K. ISHII, Localization of eigenstates and transport phenomena in one-dimensional disordered systems, Suppl. Prog. Theoret. Phys. 53 (1973), 77 -138. 393

[11] S. KOTANI, "Ljapunov indices determine absolutely continuous spectra of stationary random one-dimensional Schrödinger operators" in Stochastic Analysis (Katata/Kyoto, 1982), North-Holland Math. Library 32, North-Holland, Amsterdam, 1984, 225-247. MR 0780760393

[12] - Jacobi matrices with random potentials taking finitely many values, Rev. Math. Phys. 1 (1989), 129-133. MR 1041533 393, 397

[13] H. KUNZ and B. SOUILLARD, Sur le spectre des opérateurs aux différences finies aléatoires, Comm. Math. Phys. 78 (1980/81), 201-246. MR 0597748393

[14] D. LENZ and P. STOLLMANN, Generic sets in spaces of measures and generic singular continuous spectrum for Delone Hamiltonians, to appear in Duke Math. J., preprint, arXiv:math-ph/0410021 398

[15] L. A. PASTUR, Spectral properties of disordered systems in one-body approximation, Comm. Math. Phys. 75 (1980), 179-196. MR 0582507393

[16] B. SIMON, Kotani theory for one-dimensional stochastic Jacobi matrices, Comm. Math. Phys. 89 (1983), 227-234. MR 0709464 393, 394

[17] - Operators with singular continuous spectrum, I: General operators, Ann. of Math. (2) 141 (1995), 131-145. MR 1314033398

Avila

Laboratoire de Probabilités et Modèles aléatoires, Université Pierre et Marie Curie-Boîte courier 188,75252-Paris Cedex 05, France; artur@ccr.jussieu.fr

\section{Damanik}

Department of Mathematics, Mathematics 253-37, California Institute of Technology, Pasadena, California 91125, USA; damanik@caltech.edu 